\title{
ON SUBGROUPS OF THE ORTHOGONAL GROUP
}

\author{
BY \\ MORIO OBATA
}

0 . Introduction. We determine in this note the connected Lie subgroups $G$ of the orthogonal group $O(n)$ of degree $n$ such that

$$
\operatorname{dim} O(n-1)>\operatorname{dim} G>\operatorname{dim} O(n-3)+\operatorname{dim} O(3) .
$$

D. Montgomery and H. Samelson $[5 ; 6]$, in this regard, have proved that if

$$
\operatorname{dim} O(n)>\operatorname{dim} G \geqq \operatorname{dim} O(n-1)
$$

then $G$ is conjugate to the standard subgroup $S O(n-1)$ except $n=4,8$ and that there does not exist a subgroup $G$ such that

$$
\operatorname{dim} O(n-1)>\operatorname{dim} G>\operatorname{dim} O(n-2)+\operatorname{dim} O(2)
$$

with a finite number of exceptions for the values of $n$. But it has not yet been made clear what values of $n$ are exceptional. This note will give an answer to the analogous problem for a wider interval of the dimension of $G$.

In $\$ 1$ we shall explain some notations and then, in $\$ 2$, a relation between irreducibility and absolute irreducibility will be discussed; especially a condition for a representation to be unitary symplectic will be given. In $\$ 3$ we shall obtain inequalities concerning the dimensions of some irreducible groups. The last $\$ 4$ concerns the reducibility of $G$ satisfying relation (0.1). The method is due much to H. C. Wang and K. Yano [8] who have determined sufficiently high dimensional subgroups of the projective group. The group $G$ will be determined as the groups which leave invariant a two-dimensional plane.

1. Preliminaries. Let $G$ be a group of complex matrices of degree $m$. If $A \in G$, we may represent it in the form $A=B+(-1)^{1 / 2} C$, where $B$ and $C$ are real matrices of degree $m$. We assign to $A$ the real matrix $A^{\prime}$ of degree $2 m$ defined by

$$
A^{\prime}=\left(\begin{array}{rr}
B & C \\
-C & B
\end{array}\right)
$$

Then the correspondence $A \rightarrow A^{\prime}$ gives an isomorphism of $G$ with a group $G^{\prime}$ of real matrices of degree $2 \mathrm{~m}$. Conversely let

$$
A^{\prime}=\left(\begin{array}{rr}
B & C \\
-C & B
\end{array}\right)
$$

be an element of $G^{\prime}$. On putting

Received by the editors September 15, 1956. 


$$
P=\frac{1}{2^{1 / 2}}\left(\begin{array}{cc}
E_{m} & E_{m} \\
(-1)^{1 / 2} E_{m} & -(-1)^{1 / 2} E_{m}
\end{array}\right),
$$

$E_{m}$ being the unit matrix of degree $m$, we have

$$
P^{-1} A^{\prime} P=\left(\begin{array}{cc}
B+(-1)^{1 / 2} C & 0 \\
0 & B-(-1)^{1 / 2} C
\end{array}\right)=\left(\begin{array}{ll}
A & 0 \\
0 & \bar{A}
\end{array}\right) .
$$

As is easily seen, if $A$ is unitary, then $A^{\prime}$ is real orthogonal and vice versa. On the basis of the above consideration $G^{\prime}$ will be called the natural real representation of $G$.

Now, throughout this note we denote by $k$ the field of real numbers and by $K$ that of complex numbers and further we adopt the following notations:

$O(n, K) \quad$ the complex orthogonal group of degree $n$, $O(n)$

$S O(n)$

$U(n)$ the (real) orthogonal group of degree $n$, $S p(n)$ the special orthogonal group of degree $n$, the unitary group of degree $n$,

2. Irreducibility and absolute irreducibility. Let $G$ be a group and $\rho_{1}$ its complex representation of degree $m$. Then the sum $\rho_{1}+\bar{\rho}_{1}$ of the representation $\rho_{1}$ and its complex conjugate $\bar{\rho}_{1}$ is equivalent to a representation $\rho$ by real matrices of degree $2 m$. If $\rho_{1}$ is irreducible and is not equivalent to a real representation, then $\rho$ is irreducible in $k$ but reducible in $K$. In case $\rho_{1}$ is unitary, $\rho$ is equivalent to a real orthogonal representation. Conversely we have the following

LeMma 1. If a real representation $\rho$ of degree $n$ of a group is irreducible in $k$ and reducible in $K$, then $n$ is even, $n=2 m$, and is equivalent to the sum $\rho_{1}+\bar{\rho}_{1}$ of a complex representation $\rho_{1}$ of degree $m$ and its complex conjugate $\bar{\rho}_{1}$. Furthermore $\rho_{1}$ is irreducible. If, moreover, $\rho$ is orthogonal, then $\rho_{1}$ is equivalent to a unitary one.

The proof has been given in detail in $[1 ; 2]$, so it is omitted.

Next, let $G$ be a group having a unitary representation $\rho$ of degree $m$ $(=2 l)$. If $\rho$ is symplectic, i.e. for all $a$ in $G$ we have

$$
{ }^{t} \rho(a) J_{l} \rho(a)=J_{l},
$$

where

$$
J_{l}=\left(\begin{array}{cc}
0 & E_{l} \\
-E_{l} & 0
\end{array}\right)
$$

$\rho$ is equivalent to its complex conjugate $\bar{\rho}$, because (2.1) implies $J_{l} \rho(a) J_{l}^{-1}$ $=\bar{\rho}(a)$. The converse problem will be answered affirmatively in Lemma 3 below. To do this we state here lemmas concerning symmetric and skew symmetric matrices with complex coefficients. 
LemMa 2. Let $S$ be a symmetric matrix of degree $m$ with complex coefficients. If the equality $\bar{S} S=E_{m}$ holds, there exists a unitary matrix $T$ such that

$$
{ }^{t} T S T=E_{m} .
$$

Proof. We decompose $S$ into the real and imaginary parts, $S=S_{1}+(-1)^{1 / 2}$ $S_{2}, S_{1}$ and $S_{2}$ being real symmetric matrices. The condition $\bar{S} S=E_{m}$ is expressed by the equalities $S_{1}^{2}+S_{2}^{2}=E_{m}$ and $S_{1} S_{2}=S_{2} S_{1}$. From the latter equality it follows that there exists a common real orthogonal matrix $T_{0}$ such that ${ }^{t} T_{0} S_{1} T_{0}$ and ${ }^{t} T_{0} S_{2} T_{0}$ are both diagonal matrices:

$$
{ }^{t} T_{0} S_{1} T_{0}=\left(\begin{array}{cccc}
s_{1}^{\prime} & & & \\
& \cdot & 0 & \\
& 0 & \cdot & \\
& & & s_{m}^{\prime}
\end{array}\right), \quad{ }^{t} T_{0} S_{2} T_{0}=\left(\begin{array}{llll}
s_{1}^{\prime \prime} & & & \\
& \cdot & 0 & \\
& 0 & \cdot & \\
& & & s_{m}^{\prime \prime}
\end{array}\right),
$$

where $s_{i}^{\prime}$ and $s_{i}^{\prime \prime}(1 \leqq i \leqq m)$ are respectively the characteristic roots of $S_{1}$ and $S_{2}$. The condition $S_{1}^{2}+S_{2}^{2}=E_{m}$ implies $s_{i}^{\prime 2}+s_{i}^{\prime \prime 2}=1(1 \leqq i \leqq m)$. On putting $s_{i}=s_{i}^{\prime}+(-1)^{1 / 2} s_{i}^{\prime \prime}$ we have

$$
S_{0}={ }^{t} T_{0} S T_{0}=\left(\begin{array}{cccc}
s_{1} & & & \\
& \cdot & 0 \\
& 0 & \cdot & \\
& & & s_{m}
\end{array}\right),\left|s_{i}\right|=1 .
$$

If we choose $t_{i}$ such that $t_{i}^{2}=s_{i}(1 \leqq i \leqq m)$, the matrix

$$
\bar{T}_{1}=\left(\begin{array}{llll}
\bar{t}_{1} & & & \\
& \cdot & 0 & \\
& 0 & \cdot & \\
& & & \bar{t}_{m}
\end{array}\right)
$$

is unitary and ${ }^{t} \bar{T}_{1} S_{0} \bar{T}_{1}=E_{m}$. The matrix $T=T_{0} \bar{T}_{1}$ is a required matrix.

In an analogous way we can prove the following lemma.

Lemma $2^{\prime}$. Let $S$ be a skew-symmetric matrix of degree $m$ with complex coefficients. If the equality $\bar{S} S=-E_{m}$ holds, then $m$ is even, $m=2 l$, and there exists a unitary matrix $T$ such that

$$
{ }^{t} T S T=J_{l} .
$$

Using the above two lemmas we shall prove the following lemma [1].

LeMma 3. Let $\rho$ be a unitary representation of degree $m$ of a group. If $\rho$ is irreducible and is equivalent to its complex conjugate $\bar{\rho}$, then $\rho$ is equivalent either to a real orthogonal representation or to a unitary symplectic one. In the latter case $m$ is even.

Proof. Let $G$ be the group having the representation $\rho$. On account of the assumed equivalence of $\rho$ and $\bar{\rho}$ there exists a nonsingular matrix $S$ such that 


$$
S \rho(a) S^{-1}=\bar{\rho}(a)
$$

for all $a$ in $G$. Since $\rho$ and $\bar{\rho}$ are unitary, (2.2) implies

$$
{ }^{t} \rho(a) S \rho(a)=S,
$$

which shows that $\rho$ leaves invariant such a bilinear form that the matrix of its coefficients is $S$.

We shall show that $S$ is either symmetric or skew-symmetric. From (2.2) it follows $\bar{S} \bar{\rho}(a) \bar{S}^{-1}=\rho(a)$, which together with (2.2) gives

$$
(\bar{S} S) \rho(a)=\rho(a)(\bar{S} S) .
$$

Since $\rho$ is irreducible, by Schur's lemma, $\bar{S} S$ is a numerical multiple $\alpha E_{m}$ of the unit matrix:

$$
\bar{S} S=\alpha E_{m},
$$

$\alpha$ being a nonzero complex number. (2.4) gives the equality

$$
S \bar{S}=\bar{\alpha} E_{m} .
$$

(2.4) and (2.5) imply $\alpha=\bar{\alpha}$, i.e. that $\alpha$ is real. Without loss of generality $\alpha$ may be assumed to be \pm 1 :

$$
\bar{S} S=S \bar{S}= \pm E_{m}
$$

Next we shall see that $S$ is unitary. Because of (2.2) the representation $\rho$ leaves invariant the Hermitian matrix $H$ defined by $H={ }^{t} \bar{S} S$, i.e.

$$
{ }^{t} \bar{\rho}(a) H \rho(a)=H .
$$

Since $\rho$ is irreducible and $H$ is always positive-definite, we conclude that $H$ is a numerical multiple $\beta E_{m}$ of the unit matrix, $\beta$ being a positive real number. From (2.6) it follows $|\beta|=1$ and then $\beta=1$, which shows that $S$ is unitary:

$$
{ }^{t} \bar{S} S=E_{m} .
$$

We distinguish two cases according to the sign of $\alpha$.

(i) $\alpha=+1$. In this case the conditions (2.6) and (2.7) imply that $S$ is symmetric, $S={ }^{t} S$. By Lemma 2 there exists a unitary matrix $T$ such that ${ }^{t} T S T=E_{m}$. It follows by an easy computation that the matrix

$$
{ }^{t}\left(T^{-1} \rho(a) T\right)\left(T^{-1} \rho(a) T\right)
$$

is the unit matrix. This means that the representation $T^{-1} \rho T$ is unitary and orthogonal, i.e. it is real orthogonal.

(ii) $\alpha=-1$. In this case the conditions (2.6) and (2.7) imply that $S$ is skew-symmetric, $S=-{ }^{t} S$. By Lemma $2^{\prime} m$ is even, $m=2 l$, and there exists a unitary matrix $T$ such that ${ }^{t} T S T=J_{l}$. Then a simple calculation shows that the equality

$$
{ }^{t}\left(T^{-1} \rho(a) T\right) J_{l}\left(T^{-1} \rho(a) T\right)=J_{l}
$$


holds good. This means that the representation $T^{-1} \rho T$ is unitary symplectic.

Lemma 4 [7]. Let $G$ be a semi-simple Lie group which is not simple. Given an absolutely irreducible real orthogonal representation $\rho$ of $G$, then $\rho$ is equivalent to the Kronecker product $\rho_{1} \otimes \rho_{2}$ of absolutely irreducible representations $\rho_{1}$ and $\rho_{2}$. Furthermore $\rho_{1}$ and $\rho_{2}$ are either both real orthogonal or both unitary symplectic.

In case $\rho$ is not orthogonal the first half of the lemma holds true if $G$ is simply connected semi-simple or $G$ is a semi-simple Lie algebra.

Proof. Because of the semi-simplicity of $G$ it is written as $G=G_{1} G_{2}$ where $G_{1}$ and $G_{2}$ are semi-simple normal subgroups of $G$ such that every element of $G_{1}$ commutes with that of $G_{2}$ and $G_{1} \cap G_{2}$ is discrete. Let us denote by $\tilde{\rho}_{i}$ the restriction of $\rho$ to $G_{i}(i=1,2)$. Since $\tilde{\rho}_{i}$ may be reducible, we decompose $\tilde{\rho}_{1}$ into irreducible components in $K$ :

$$
\tilde{\rho}_{1} \approx \rho_{1}+\rho_{1}^{\prime}+\cdots+\rho_{1}^{\left(n_{2}-1\right)} .
$$

From the facts that $\rho_{1}^{(i)}$ is irreducible in $K$ and that every element of $G_{2}$ commutes with that of $G_{1}$ it follows that $\rho_{1}, \rho_{1}^{\prime}, \cdots, \rho_{1}^{\left(n_{2}-1\right)}$ are of the same degree $n_{1}$ and are equivalent to each other. Thus there exists a nonsingular matrix $P$ such that

$$
P^{-1} \tilde{\rho}_{1} P=\rho_{1}+\cdots+\rho_{1} \text { ( } n_{2} \text { components). }
$$

Again by irreducibility of $\rho_{1}$ and commutativity of $G_{2}$ with $G_{1}$, for every $a_{2}$ in $G_{2}, P^{-1} \widetilde{\rho}_{2}\left(a_{2}\right) P$ is of the form

$$
P^{-1} \tilde{\rho}_{2}\left(a_{2}\right) P=\left(\begin{array}{ccc}
b_{11} E_{n_{1}} & \cdots & b_{1 n_{2}} E_{n_{1}} \\
\vdots & & \vdots \\
b_{n_{2} 1} E_{n_{1}} & \cdots & b_{n_{2} n_{2}} E_{n_{1}}
\end{array}\right)
$$

On putting

$$
\rho_{2}\left(a_{2}\right)=\left(\begin{array}{ccc}
b_{11} & \cdots & b_{1 n_{2}} \\
\vdots & \vdots \\
\dot{b_{n_{2} 1}} & \cdots & \dot{b_{n_{2} n_{2}}}
\end{array}\right),
$$

we find that the representation $\rho_{2}$ is irreducible in $K$ and $P^{-1} \widetilde{\rho}_{2} P=E_{n_{1}} \otimes \rho_{2}$ and then

$$
P^{-1} \rho P=\rho_{1} \otimes \rho_{2} .
$$

It should be noted that the relationship of $\rho_{1}$ and $\rho_{2}$ is mutual.

To prove the second half of this lemma, we first decompose $\tilde{\rho}_{1}$ into irreducible components in $k$. We distinguish two cases.

(i) In case each of the components is absolutely irreducible, by using this decomposition we have 


$$
P^{-1} \rho P=\rho_{1} \otimes \rho_{2} .
$$

Since the decomposition of $\tilde{\rho}_{1}$ is done in $k, \rho_{1}$ and $P$ may be assumed to be real orthogonal, so also is $\rho_{2}$ by $(2.10)$. Thus in this case $\rho$ is equivalent to the Kronecker product of two real orthogonal representations.

(ii) In case some one of the components of $\tilde{\rho}_{1}$ is reducible in $K$, we shall prove that all of the components are reducible in $K$ and the irreducible components in $K$ are unitary symplectic.

To prove these, let

$$
P_{1}^{-1} \rho_{1} P_{1}=\sigma_{1}+\cdots+\sigma_{p}
$$

be the decomposition of $\tilde{\rho}_{1}$ into irreducible components in $k$, where $P_{1}$ may be assumed to be real orthogonal. We assume that $\sigma_{1}, \cdots, \sigma_{q}$ are reducible in $K$ but $\sigma_{q+1}, \cdots, \sigma_{p}$ are absolutely irreducible. By Lemma $1 \sigma_{i}(1 \leqq i \leqq q)$ is equivalent to the sum $\sigma_{i}^{\prime}+\bar{\sigma}_{i}^{\prime}$ of a unitary representation $\sigma_{i}^{\prime}$ and its complex conjugate $\bar{\sigma}_{i}^{\prime}$, where $\sigma_{i}^{\prime}$ can not be equivalent to a real one. Since the decompositions $\sigma_{i}$ into $\sigma_{i}^{\prime}+\bar{\sigma}_{i}^{\prime}(1 \leqq i \leqq q)$ are realized by unitary matrices, there exists a unitary matrix $P_{2}$ such that

$$
P_{2}^{-1} \tilde{\rho}_{2} P_{1}=\sigma_{1}^{\prime}+\bar{\sigma}_{1}^{\prime}+\cdots+\sigma_{q}^{\prime}+\bar{\sigma}_{q}^{\prime}+\sigma_{q+1}+\cdots+\sigma_{p} .
$$

As we have seen above that all the components of this decomposition are equivalent to each other, $\sigma_{1}^{\prime}$ must be equivalent to the real orthogonal $\sigma_{j}$ $(q+1 \leqq j \leqq p)$, which leads to a contradiction. Thus we have

$$
\tilde{\rho}_{1} \approx \sigma_{1}^{\prime}+\bar{\sigma}_{1}^{\prime}+\cdots+\sigma_{p}^{\prime}+\bar{\sigma}_{p}^{\prime} \quad\left(2 p=n_{2}\right) .
$$

Since $\sigma_{1}^{\prime}$ is equivalent to its complex conjugate $\bar{\sigma}_{1}^{\prime}$, by Lemma $3 \sigma_{1}^{\prime}$ is equivalent to a unitary symplectic representation $\rho_{1}$. Since the equivalence can be realized by a unitary matrix, from the first part of this lemma there exist a unitary matrix $P$ and a unitary representation $\rho_{2}$ of degree $n_{2}$ such that

$$
P^{-1} \tilde{\rho}_{1} P=\rho_{1}+\cdots+\rho_{1} \quad\left(n_{2} \text { components }\right) \quad \text { and } \quad P^{-1} \rho P=\rho_{1} \otimes \rho_{2} .
$$

The above consideration shows that $\rho_{1}$ is either real orthogonal or unitary symplectic, so also is $\rho_{2}$ because the relation of $\rho_{1}$ and $\rho_{2}$ is mutual. Since we have seen in (i) that if $\rho_{1}$ is orthogonal so is $\rho_{2}$, we conclude that in case $\rho_{1}$ is unitary symplectic so also is $\rho_{2}$, which completes the proof.

3. The dimensions of irreducible subgroups of the orthogonal group. We obtain in this section, using the results of the preceding section, inequalities concerning the dimensions of irreducible subgroups of the orthogonal group.

Lemma 5. Let $G$ be an irreducible group of real orthogonal matrices of degree n. If $G$ is reducible in $K$, then the inequality

$$
\operatorname{dim} G \leqq n^{2} / 4
$$

holds good. 
Proof. In view of the assumed irreducibility in $k$ and reducibility in $K$, by Lemma $1 n$ is even, $n=2 m$, and there exists a nonsingular matrix $T$ such that for all $A$ in $G$

$$
T^{-1} A T=\left(\begin{array}{cc}
B & 0 \\
0 & \bar{B}
\end{array}\right),
$$

$B$ being a unitary matrix. This shows that $G$ is the natural real representation of a subgroup of the unitary group $U(m)$. Hence we have

$$
\operatorname{dim} G \leqq \operatorname{dim} U(m)=n_{2} / 4 .
$$

Next, as a condition for a Lie algebra to be semi-simple we have the following lemma due to E. Cartan [3, p. 147].

LemMa 6. Let $g$ be a Lie algebra of complex matrices of degree $n$. If $g$ is irreducible, then it is either semi-simple or equivalent to the direct sum of a semisimple Lie algebra and the Lie algebra consisting of all the matrices of the form $c E_{n}, c$ being a complex number. If, in addition, $g$ is contained in the Lie algebra of the complex orthogonal group $O(n, K)$, then $g$ is semi-simple.

The proof is omitted.

Lemma 7. Let $G$ be an absolutely irreducible Lie group of real orthogonal matrices of degree $n$. If $G$ is not simple, then the inequality

$$
\operatorname{dim} G \leqq\left(n^{2}+2 n\right) / 4
$$

holds good.

Proof. We denote by $r$ the dimension of $G$. Since $G$ is semi-simple by Lemma 6 and is not simple by hypothesis, it is written as $G=G_{1} G_{2}$ as in the proof of Lemma 4, where we may assume without loss of generality

$$
\operatorname{dim} G_{1} \geqq \operatorname{dim} G_{2} \text { or } \operatorname{dim} G_{1} \geqq r / 4 .
$$

By Lemma 4 there exist representations $\rho_{i}$ of degree $n_{i}(i=1,2)$ such that the faithful representation $\rho: A \rightarrow A(A \in G)$ is equivalent to $\rho_{1} \otimes \rho_{2}$. As is easily seen, $\rho_{i}$ is a faithful representation of $G_{i}(i=1,2)$ provided that $\rho$ is faithful in the proof of Lemma 4 . Thus the matric group $G$ is equivalent to $\widetilde{G}_{1} \otimes \widetilde{G}_{2}$ where $\widetilde{G}_{i}=\rho_{i}\left(G_{i}\right)$. Since $n_{1} n_{2}=n$ and $n_{i} \geqq 2$, we have

$$
n_{i} \leqq n / 4 \text {. }
$$

Since by Lemma $4 \rho_{i}$ may be assumed to be either a real orthogonal representation or a unitary symplectic one, $\tilde{G}_{i}$ is contained in $O\left(n_{i}\right)$ or $S p\left(n_{i} / 2\right)$. From the facts that

$$
\operatorname{dim} O\left(n_{i}\right)=\frac{1}{2} n_{i}\left(n_{i}-1\right) \text { and } \operatorname{dim} S p\left(n_{i} / 2\right)=\frac{1}{2} n_{i}\left(n_{i}+1\right)
$$


we have in both cases

$$
\operatorname{dim} G_{i}=\operatorname{dim} \tilde{G}_{i} \leqq \frac{1}{2} n_{i}\left(n_{i}+1\right)
$$

because $n_{i}\left(n_{i}-1\right) / 2<n_{i}\left(n_{i}+1\right) / 2$. From (3.1), (3.2) and (3.3) it follows

$$
\frac{r}{2} \leqq \frac{n}{4}\left(\frac{n}{2}+1\right) \text { or } r \leqq \frac{1}{4}\left(n^{2}+2 n\right) \text {. }
$$

LEMMA 8. Let $g$ be an absolutely irreducible Lie algebra of real matrices of degree $n$ and $g^{K}$ the complex form of $g$. If $g$ is simple but $g^{K}$ is not simple, then the inequality

$$
\operatorname{dim} g \leqq 2(n-1)
$$

holds good.

Proof. We put $\operatorname{dim} g=r$. Since the semi-simplicity is an implication of the fact that the fundamental quadratic form is non-degenerate, $g$ is semi-simple. $g$, however, being simple but $g^{K}$ being not simple, $g^{K}$ is the direct sum of two complex conjugate ideals $h$ and $\bar{h}$, each of which is simple and of complex dimension $r / 2$. Then in the same manner as in Lemma $4 g^{K}$ can be written as the Kronecker product of two matric Lie algebras $h^{*}$ and $\bar{h}^{*}$ of degree $n_{1}$ and $n_{2}$ respectively, $h^{*}$ and $\bar{h}^{*}$ being isomorphic images of $h$ and $\bar{h}$ respectively. It follows

$$
\operatorname{dim} h=\operatorname{dim} h^{*}=r / 2 \leqq n_{1}^{2}-1 \text { and } \operatorname{dim} \bar{h} \leqq n_{1}^{2}-1 .
$$

These imply $r \leqq 2(n-1)$ because $n_{1} n_{2}=n$.

4. Reducibility of subgroups of the orthogonal group. We first prove

LEMMA 9. Let $G$ be a connected Lie group of real orthogonal matrices of degree $n$. If $n \geqq 13$ and

$$
\operatorname{dim} O(n)>\operatorname{dim} G \geqq \operatorname{dim} O(n-3)+\operatorname{dim} O(3)+1=\frac{1}{2}\left(n^{2}-7 n+20\right),
$$

then $G$ is reducible in $k$.

Proof. Suppose that $G$ is irreducible in $k$ and we shall show that this leads to a contradiction.

If $G$ is irreducible, $G$ is absolutely irreducible. In fact, if $G$ is reducible in $K$, then by Lemma 5 we have $\operatorname{dim} G \leqq n^{2} / 4$. But this can not occur because the inequality

$$
\operatorname{dim} G \geqq \frac{1}{2}\left(n^{2}-7 n+20\right)>\frac{n^{2}}{4}
$$


holds for $n \geqq 11$. Thus $G$ must be absolutely irreducible provided that $G$ is irreducible in $k$. Then the complex form $G^{K}$ of $G$ is also irreducible. It follows from Lemma 6 that $G^{K}$ is semi-simple, so also is $G$.

We distinguish two cases.

(i) $G$ is not simple. In this case by Lemma 7 we must have $\operatorname{dim} G$ $\leqq\left(n^{2}+2 n\right) / 4$. But by hypothesis we have for $n \geqq 13$

$$
\operatorname{dim} G \geqq \frac{1}{2}\left(n^{2}-7 n+20\right)>\frac{1}{4}\left(n^{2}+2 n\right) .
$$

Thus this case can not occur.

(ii) $G$ is simple. Since $\operatorname{dim} G \geqq\left(n^{2}-7 n+20\right) / 2>2(n-1)$ for $n \geqq 13, G^{K}$ is also simple by Lemma 8 . Denoting by $g$ the Lie algebra of $G^{K}, g$ is one of the simple Lie algebras in the classification of E. Cartan [2, p. 147]. Since $g$ is contained in the Lie algebra of the orthogonal group $O(n, K)$, if we denote by $p$ the rank of the former algebra and by $q$ that of the latter, we have

$$
p \leqq q=\left[\frac{n}{2}\right] \text {. }
$$

On account of the assumptions $n \geqq 13, n(n-1) / 2>\operatorname{dim} G \geqq\left(n^{2}-7 n+20\right) / 2$ and $p \leqq q$, the possible types of $g$ are the following.

CASE I. $n=2 q+1, g \subset B_{q}$.

Type of $g \quad$ Rank of $g$

$q-1$
$q-1$
$q$
6
7
7
8
8
4

Dimension of $g$ Rank of $B_{q}$

$$
\begin{aligned}
& (n-2)(n-3) / 2 \\
& (n-2)(n-3) / 2 \\
& (n-1)(n-2) / 2
\end{aligned}
$$

72

133

$q$
$q$
$q$
6
8
9
11
12
6

$n$

$2 q+1$

$2 q+1$

$2 q+1$

13

17

19

23

25

13

Case II. $n=2 q, g \subset D_{q}$.

Type of $g$

(E)
Rank of $g$

$$
q-1
$$$$
q-1
$$$$
q-1
$$

6

7

8
Dimension of $g$ Rank of $D_{q}$

$(n-1)(n-2) / 2$

$(n-1)(n-2) / 2$

$(n-2)(n-3) / 2$

72

133

248 $q$

$q$

$q$

7

9

12 $n$ $2 q$ $2 q$ $2 q$ 14 18 24 
But the theory of representations of complex simple Lie algebras tells us that in both cases I and II all these Lie algebras do not have irreducible representations of the corresponding degree $n$, so we may conclude that $g$ can not be simple, which shows that the case (ii) can not occur.

Thus in all cases we have reached contradictions, so that $G$ must be reducible in $k$.

We can now determine the explicit form of the group $G$ for $n \geqq 14$.

THEOREM. Let $G$ be a connected Lie subgroup of the orthogonal group $O(n)$ of degree $n$. We assume that $n \geqq 14$ and

$$
\operatorname{dim} O(n-1)>\operatorname{dim} G>\operatorname{dim} O(n-3)+\operatorname{dim} O(3) .
$$

Then $G$ leaves invariant one and only one plane, i.e. $G$ consists of all the matrices of the form

$$
\text { either }\left(\begin{array}{ll}
E_{2} & 0 \\
0 & A
\end{array}\right), A \in S O(n-2) \text { or }\left(\begin{array}{ll}
B & 0 \\
0 & C
\end{array}\right), B \in S O(2), C \in S O(n-2)
$$

up to a conjugation in $O(n)$.

Proof. Since the assumptions of Lemma 9 are satisfied, $G$ is reducible in $k$. If we denote by $V$ the $n$-dimensional vector space in $k$ acted on by $G$, then $V$ is written as the direct sum $V_{1}+V_{2}$ of invariant subspaces $V_{1}$ and $V_{2}$. On putting $\operatorname{dim} V_{1}=m$, we have $\operatorname{dim} V_{2}=n-m$ and

$$
\operatorname{dim} G \leqq \operatorname{dim} O(m)+\operatorname{dim} O(n-m) .
$$

We may assume without loss of generality $m \leqq n-m$. On account of the inequality $\operatorname{dim} G>\operatorname{dim} O(3)+\operatorname{dim} O(n-3)$ we have $m=1$ or $m=2$.

CASE I. $m=1$. By connectedness of $G$ every element of $V_{1}$ is remained invariant under all the transformations of $G$. Accordingly every element $A$ of $G$ is of the form

$$
\left(\begin{array}{cc}
1 & 0 \\
0 & A^{\prime}
\end{array}\right), \quad A^{\prime} \in S O(n-1)
$$

up to an inner automorphism of $O(n)$. The image $G^{\prime}$ of $G$ by the isomorphism $A \rightarrow A^{\prime}$ is a connected Lie subgroup of $O(n-1)$ and satisfies the inequality

$$
\operatorname{dim} O(n-1)>\operatorname{dim} G^{\prime}>\operatorname{dim} O(3)+\operatorname{dim} O(n-3), \quad n-1 \geqq 13 .
$$

By Lemma $9 G^{\prime}$ must be also reducible on $V_{2}$ in $k$, so $V_{2}$ is the direct sum $U_{1}+U_{2}$ of invariant subspaces $U_{1}$ and $U_{2}$, where we may assume $\operatorname{dim} U_{1}$ $\leqq \operatorname{dim} U_{2}$. Then $\operatorname{dim} U_{1}$ must be 1 and again by connectedness of $G^{\prime}$ every element $A^{\prime}$ of $G^{\prime}$ is of the form

$$
\left(\begin{array}{cc}
1 & 0 \\
0 & A^{\prime \prime}
\end{array}\right), \quad A^{\prime \prime} \in S O(n-2)
$$


up to a conjugation in $O(n-1)$. The image $G^{\prime \prime}$ of $G^{\prime}$ by the isomorphism $A^{\prime} \rightarrow A^{\prime \prime}$ is a connected Lie subgroup of $O(n-2)$. Since $\operatorname{dim} G^{\prime \prime}>\operatorname{dim} O(n-3)$ $G^{\prime \prime}$ must coincide with $S O(n-2)$. Thus $G$ consists of all the matrices of the form

$$
\left(\begin{array}{ll}
E_{2} & 0 \\
0 & A^{\prime \prime}
\end{array}\right), \quad A^{\prime \prime} \in S O(n-2)
$$

up to a conjugation in $O(n)$.

CASE II. $m=2$. In this case we may assume that $G$ is irreducible in $V_{1}$. In fact, if not, the case reduces to Case I. Furthermore $G$ may be assumed to consist of matrices of the form

$$
\left(\begin{array}{ll}
A & 0 \\
0 & B
\end{array}\right), \quad A \in O(2), B \in O(n-2) .
$$

Let us denote by $G_{1}$ and $G_{2}$ the subgroups of $G$ consisting of all the matrices which leave fixed all elements of $V_{2}$ and $V_{1}$ respectively. Then if $A_{1} \in G_{1}$ and $A_{2} \in G_{2}$ they are written in the forms

$$
A_{1}=\left(\begin{array}{cc}
A^{\prime} & 0 \\
0 & E_{n-2}
\end{array}\right), \quad A^{\prime} \in O(2), \quad A_{2}=\left(\begin{array}{cc}
E_{2} & 0 \\
0 & A^{\prime \prime}
\end{array}\right), \quad A^{\prime \prime} \in O(n-2) .
$$

It is evident that $G_{1} \cap G_{2}$ consists of the unit matrix $E_{n}$ only and every element of $G_{1}$ commutes with that of $G_{2}$. We shall see that $G$ is the direct product of $G_{1}$ and $G_{2}$. To do this it is sufficient to prove $G_{1} G_{2}=G$.

Since $G^{\prime \prime}=G / G_{1}$ is contained in $O(n-2)$ in $V_{2}$, from the inequality

$$
\operatorname{dim} G^{\prime \prime} \geqq \operatorname{dim} G-1>\operatorname{dim} O(n-3)
$$

it follows that $G^{\prime \prime}$ is the special orthogonal group $S O(n-2) . G^{\prime}=G / G_{2}$ is irreducible in $V_{1}$, so it is $S O(2)$. It follows $\operatorname{dim} G_{2}=\operatorname{dim} G-1$. Since $G_{2}$ $=G_{2} /\left(G_{1} \cap G_{2}\right)$ is regarded as a subgroup of $G^{\prime \prime}$ and

$$
\operatorname{dim} G_{2}=\operatorname{dim} G-1>\operatorname{dim} O(n-3),
$$

$G_{2}$ coincides with $G^{\prime \prime}=S O(n-2)$. Thus we have an isomorphism $G_{2} \rightarrow G / G_{1}$ and this is obviously canonical. Therefore we have $G=G_{1} G_{2}$. On the other hand it can be proved that $G_{1}$ is isomorphic with $S O(2)$. Thus the direct product $G$ of $G_{1}$ and $G_{2}$ consists of all the matrices of the form

$$
\left(\begin{array}{ll}
A & 0 \\
0 & B
\end{array}\right), \quad A \in S O(2), B \in S O(n-2) \text {. }
$$

\section{BIBLIOGRAPHY}

1. M. Abe, Irreduzibilität und absolute Irreduzibilität des Matrizensystems, Proceedings of the Physico Mathematical Society of Japan vol. 24 (1942) pp. 769-789. 
2. E. Cartan, Sur la structure de groupes de transformations finis et continus, Thèse, Paris, 1894.

3. - Les groupes de transformations continus, infinis, simples, Ann. École Norm. Sup. vol. 26 (1909) pp. 93-161.

4. H. Iwamoto, On the structure of Riemannian spaces whose holonomy groups fix a nullsystem, Tôhoku Math. J. vol. 1 (1949) pp. 109-135.

5. D. Montgomery and H. Samelson, Transformation groups of spheres, Ann. of Math. vol. 44 (1943) pp. 454-470.

6. M. Obata, On n-dimensional homogeneous spaces of Lie groups of dimension greater than $n(n-1) / 2$, Journal of the Mathematical Society of Japan vol. 7 (1955) pp. 371-388.

7. S. Sasaki, On the influence of the topological structure of Riemannian manifolds upon their holonomy groups, Tôhoku Math. J. vol. 6 (1954) pp. 135-148.

8. H. C. Wang and K. Yano, A class of affinely connected spaces, Trans. Amer. Math. Soc. vol. 80 (1955) pp. 72-92.

Tokyo Metropolitan University, Setagaya, Tokyo, Japan 